Corrigendum

\title{
Corrigendum to "Spino-cerebellar tDCS modulates N100 components of the P300 event related potential" [Neuropsychologia 135 (2019) 107231]
}

\author{
Fabiana Ruggiero $^{\text {a }}$, Roberta Ferrucci ${ }^{\text {a,b,c }}{ }^{\text {, Tommaso Bocci }}{ }^{\text {b,c }}$, Martina Nigro ${ }^{\text {a }}$, \\ Maurizio Vergari ${ }^{\text {a, }}$, Sara Marceglia ${ }^{\mathrm{a}, \mathrm{d}}$, Sergio Barbieri ${ }^{\mathrm{a}}$, Alberto Priori ${ }^{\mathrm{b}, \mathrm{c}, *}$ \\ a Foundation IRCCS Ca' Granda Ospedale Maggiore Policlinico, Neurophysiology Unit, Milan, Italy \\ b "Aldo Ravelli" Center for Neurotechnology and Experimental Brain Therapeutics, Dipartimento di Scienze Della Salute, Universita Degli Studi di Milano, Milan, Italy \\ ${ }^{\mathrm{c}}$ III Neurology Clinic, ASST Santi Paolo e Carlo, Milan, Italy \\ ${ }^{\mathrm{d}}$ Dipartimento di Ingegneria e Architettura, University of Trieste, Trieste, Italy
}

The authors would like to provide the readership of Neuropsychologia with a reference that was missing from the original publication of our research article, namely: Bianco, V., Di Russo, F., Perri, R.
L., \& Berchicci, M. (2017). Different proactive and reactive action control in fencers' and boxers' brain. Neuroscience, 343, 260-268.

The authors would like to apologise for any inconvenience caused.

DOI of original article: https://doi.org/10.1016/j.neuropsychologia.2019.107231.

* Corresponding author. "Aldo Ravelli” Center for Neurotechnology and Experimental Brain Therapeutics, Dipartimento di Scienze Della Salute, Universita Degli Studi di Milano, Milan, Italy

E-mail address: alberto.priori@unimi.it (A. Priori).

https://doi.org/10.1016/j.neuropsychologia.2019.107310

Available online 11 January 2020

0028-3932/@ 2019 Published by Elsevier Ltd. 